Bull. Mater. Sci., Vol. 6, No. 6, December 1984, pp. 1047-1052. (C) Printed in India.

\title{
Evaluation of magnetic hyperfine field distributions from Mössbauer spectra of disordered alloys and metallic glasses
}

\author{
C BANSAL \\ School of Physics, University of Hyderabad, Hyderabad 500134 , India \\ MS received 15 March 1983
}

Abstract. A simple computer program is described to evaluate magnetic field distributions
from complex Mössbauer spectra of magnetic alloys. The use of this program for test cases of
Vitrovac 4040 amorphous sample and a simulated spectrum of two gaussian peaks is discussed.

Keywords. Mössbauer spectra; metallic glasses; magnetic hyperfine field distributions.

\section{Introduction}

The Mössbauer spectra for disordered magnetic alloys and metailic glasses are generally complex because of the overlap of the component lines due to a wide variety of environments in which the Mössbauer atom is situated. In some cases when the overlap is not too large the average hyperfine field can still be evaluated if the field is distributed around an average value (Bansal et al 1975). In other cases when the broadening is large this may not be possible. When two or more types of magnetic behaviour are present i.e. magnetic splitting due to magnetically ordered regions and a single line due to a paramagnetic contributions it is again very difficult to extract useful information from Mössbauer spectra (Bansal and Chandra 1979).

Several methods have been proposed to obtain the distribution of magnetic fields which generate the complex spectra (Window 1971; Hesse and Rübartsch 1974; Logan and Sun 1976) and improvements on them also given (LaCaer and Dubois 1979; Wivel and Morup 1981).

\section{Window's method}

Amongst these, the method due to Window is a model-independent field distribution analysis which assumes an arbitrary probability distribution of the magnetic hypertine fields $P(H)$ and expands it in a Fourier series of trignometric functions.

$$
P(H)=\sum_{n=1}^{N} a_{n}\left[\cos \left(n \pi H / H_{\max }\right)-\cos n \pi\right] .
$$

The field distribution in this form satisfies the boundary conditions: (i) $P(-H)$ $=P(H)$, since in the absence of an external field the lines produced due to a field $H$ are in the same positions as those produced by a field $-H$. The series therefore contains 
only cosine terms. (ii) There is an upper cut-off point beyond which $P(H)$ goes to zero smoothly i.e.

$$
\left.\frac{\mathrm{d} P(H)}{\mathrm{d} H}\right]_{H=H_{\max }}=0 .
$$

If now the coefficients $a_{n}$ of the series can be determined so as to fit the experimental spectrum, the required field distribution is known.

The Mössbauer spectrum generated at velocity $v$ due to this distribution is given by

$$
S(v)=\int_{0}^{H_{\max }} P(H) L_{6}(v, H) \mathrm{d} H,
$$

where

$$
L_{6}(v, H)=\sum_{i=1}^{6} \frac{1}{\pi} \frac{\Gamma_{i} / 2}{\left(\Gamma_{i} / 2\right)^{2}+\left(v-\alpha_{i} H\right)^{2}},
$$

is the normalised Lorentzian shape function of the lines of a component spectrum for field $H . \Gamma_{i}$ are the linewidths of the six lines of the component spectrum and $\alpha_{i} H$ are their positions. Equation (2) can be rewritten as

$$
\begin{aligned}
& S(v)=\sum a_{n} \int_{0}^{H_{\max }} f_{n}(H) L_{6}(v, H) \mathrm{d} H, \\
& =\sum_{n} a_{n} S_{n}(v), \\
& \text { where } \quad S_{n}(v)=\int_{0}^{H_{\max }} f_{n}(H) L_{6}(v, H) \mathrm{d} H
\end{aligned}
$$

is the spectrum created by the term $f_{n}(H)$. If the experimental spectrum at velocity $v$ is denoted by $W(v)$, the coefficients $a_{n}$ of the field distribution can be found by minimizing the function

$$
\phi=\sum_{v}[S(v)-W(v)]
$$

with respect to the parameters $a_{n}$ or other parameters like isomer shift if necessary. A $\chi^{2}$ nonlinear least square fitting procedure is normally used to obtain the required coefficient $a_{n}$. This procedure is iterative and therefore time-consuming. If the isomer shift is also required to be obtained from a fit to the spectrum this procedure is justified. However in most systems when one is not concerned with relatively small isomer shift values exactly one can safely assume a constant isomer shift for all the elementary fields $H$. A good guess for this can usually be obtained from the centroid of the spectrum or from a spectrum above the Curie temperature. This then can be given as a fixed parameter. 


\section{Procedure}

We get $N$ simultaneous equations which are linear in $a_{n}$ if we differentiate $\phi$ in (4), with respect to $a_{n}$ 's and set them equal to zero.

$$
\begin{aligned}
\phi & =\sum_{v}\left[\sum_{n} a_{n} S_{n}(v)-W(v)\right]^{2}, \\
\frac{1}{2} \frac{\partial \phi}{\partial a_{n}} & =\sum_{v}[S(v)-W(v)] S_{n}(v) .
\end{aligned}
$$

Differentiating once again w.r.t $a_{m}$

$$
\frac{1}{2} \frac{\partial^{2} \phi}{\partial a_{m} \partial a_{n}}=\sum_{v} S_{m}(v) S_{n}(v)=X_{m n} \text { (say). }
$$

Substituting (7) in (6) and setting $\partial \phi / \partial a_{n}=0$

$$
\sum_{m} a_{m} X_{m n}-\sum_{v} W(v)=0
$$

There are $n$ such equations for $n=1,2 \ldots N$.

Defining the matrices

$$
\begin{aligned}
\beta_{n} & =\left[\sum_{v} W(v) S_{n}(v)\right], \\
X_{m n} & =\left[\sum_{v} S_{m}(v) S_{n}(v)\right],
\end{aligned}
$$

we get the matrix equation

$$
a X=\beta \text {. }
$$

This can be solved to get the matrix of the coefficients $a_{n}$

$$
a=X^{-1} \beta \text {. }
$$

A simple computer program can be written to set up the matrices $X$ and $\beta$, finding the inverse of $X$ and then calculating $a$. These coefficients can then be used to generate the field distribution $P(H)$.

\section{Examples of use}

A test usage of the program is illustrated in figures 1 and 2. In figure 1, spectrum $A$ is the Mössbauer spectrum simulated for two Gaussian fields of equal intensity as shown in $A^{\prime}$. The intensity ratios of the component spectra were constrained in the ratio $3: 2: 1: 1: 2: 3$ and the isomer shift fixed at zero. Using 5 terms in the fourier series expansion of $P(H)$ the field distribution obtained using the program outlined above is shown in $\mathrm{B}^{\prime}$. The two peaks are unresolved. When 10 terms are included in the Fourier series the distribution marked $\mathrm{C}^{\prime}$ is obtained and the simulated and fitted spectrum agree very well as shown in $\mathrm{C}$.

Figure 2 shows the experimental Mössbauer spectrum for an amorphous sample (Vitrovac 4040 from Vacuumschmelze GMBH, Hanau, Germany) together with the 


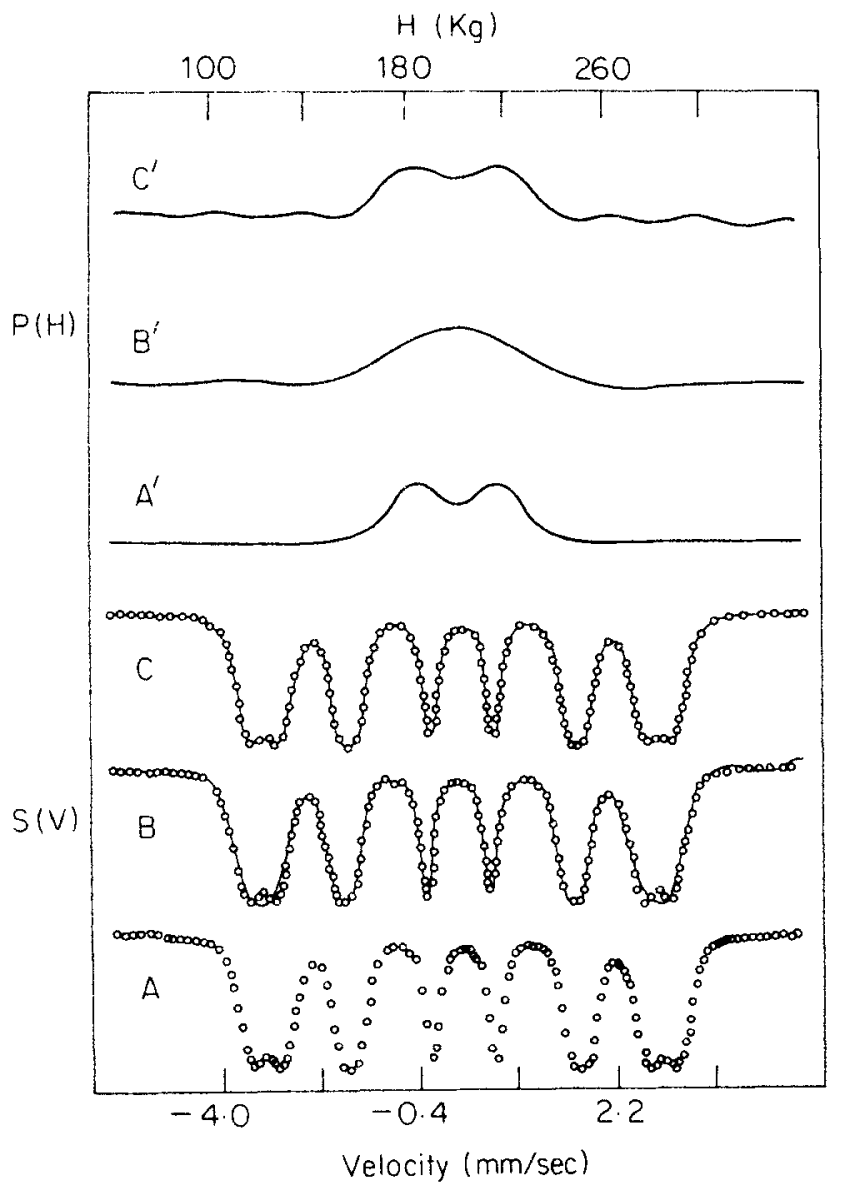

Figure 1. The Mössbauer spectrum A is simulated for two Gaussian peaks shown in A'. The field distribution $B^{\prime}$ is obtained using the computer program described here when 5 terms are used in the Fourier series expansion of $P(H)$. The corresponding Mössbauer spectrum is shown in $B$. For 10 terms in $P(H)$ the distribution $C^{\prime}$ is obtained and the corresponding fit is shown in $\mathrm{C}$.

field distributions obtained using this program. In fitting such spectra there is uncertainty in the value of the intensity ratios to be chosen for the elementary spectra. One of the ways generally used is to vary the intensity ratio in the form $3: x: 1: 1: x: 3$ and use that value of $x$ which gives the best fit to the experimental spectrum. The field distributions for various $x$ values are shown in figure 2. For a value $x=2.5$ one gets a good fit to the spectrum.

The number of terms in the Fourier series that should be retained in the expansion for $P(H)$ also has to be chosen carefully. For a small number of terms the resolution is poor. As seen in figure 1, the two Gaussian peaks are not resolved when only 5 terms are used but are seen separately for 10 terms in the expansion. However if the number of terms is made very large there occurs another difficulty. The distribution tries to fit the statistical fluctuation in data which are unphysical and thereby produces oscillations in the field distribution. 


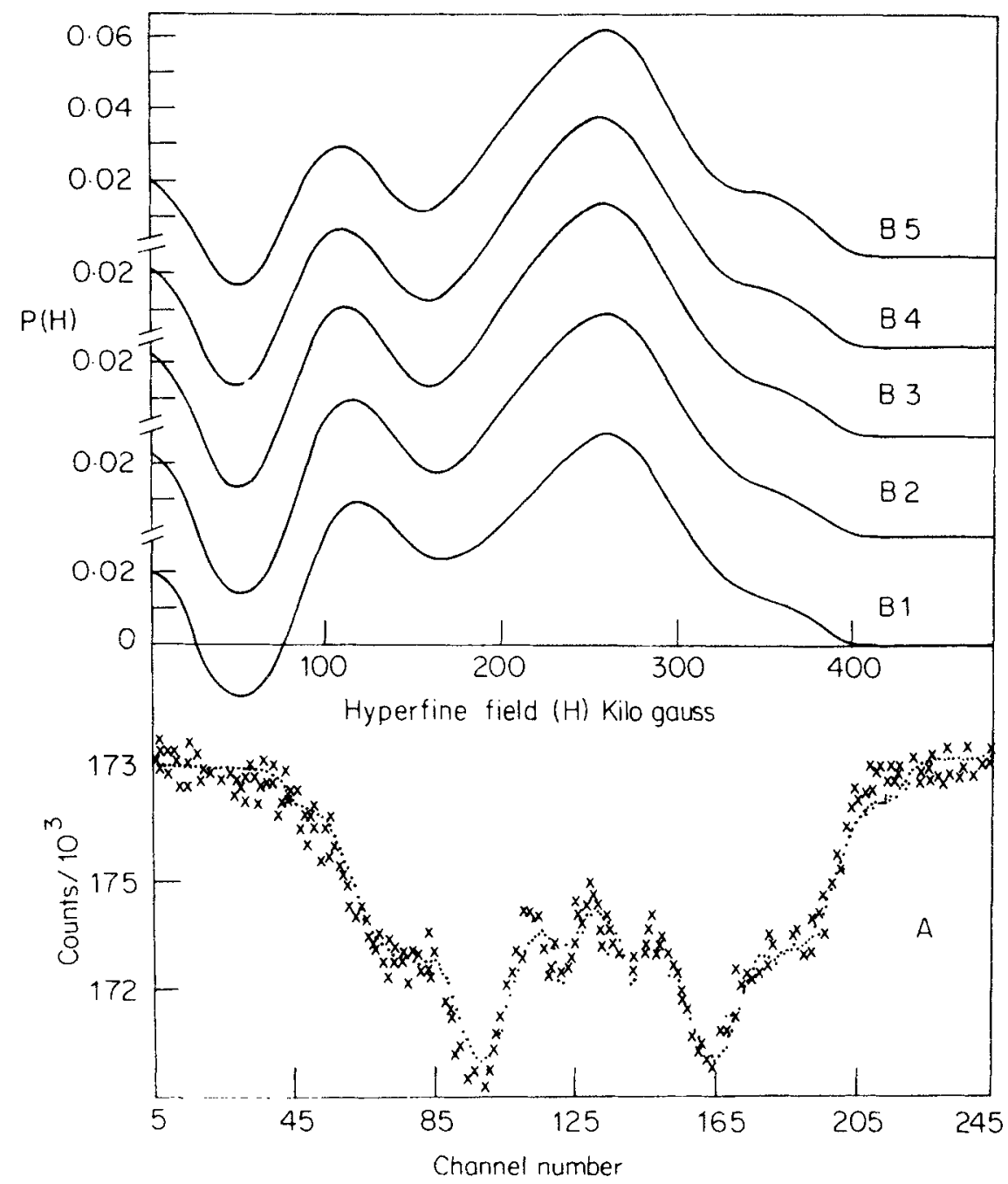

Figure 2. The experimental Mössbauer spectrum for Vitrovac 4040 sample $(+)$ and fitted spectrum $(\cdot)$ for the field distribution in B2. The field distributions for various values of $x$ (intensity ratio of 2 nd and 5 th lines relative to $3 \mathrm{rd}$ and 4 th lines) are shown as B1 $(x=2), \mathrm{B} 2$ ( $x$ $=2 \cdot 5), \mathrm{B} 3(x=3), \mathrm{B} 4(x=3 \cdot 5), \mathrm{B} 5(x=4)$.

\section{Conclusions}

The program described above is simple to write and can be simply modified to analyse spectra which have a paramagnetic single line in addition to distributions at higher velocities or for situations in which the baseline is parabolic. A listing of the program is available with us and has about 175 statements in Fortran IV.

\section{Acknowledgement}

The author is grateful to Dr R G Pillay of the TIFR. Bombay, for help in writing the program and testing it. 


\section{References}

Bansal C, Ray J and Chandra G 1975 J. Phys. F5 1663

Bansal C and Chandra G 1979 Phys. Rev. B20 3357

Hesse J and Rübartsch A 1974 J. Phys. E7 526

LaCaer G and Dubois J M (1979) J. Phys. E12 1083

Logan J and Sun E 1976 J. Noncryst. Solids 20285

Window B 1971 J. Phys. E4 401

Wivel C and Morup S 1981 J. Phys. E14 605 